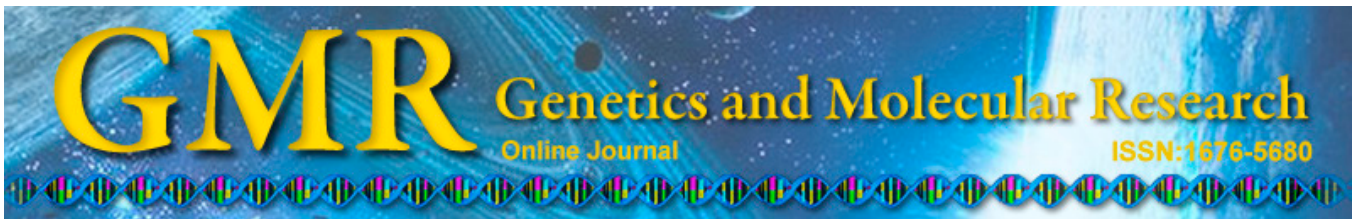

\title{
Identification and expression analysis of cDNA encoding chitinase-like protein $(C L P)$ gene in Japanese scallop Mizuhopecten yessoensis
}

\author{
L. Gao ${ }^{1}$, G.J. Xu' ${ }^{2}$, H. Su${ }^{1}$, X.G. Gao ${ }^{1}$, Y.F. Li ${ }^{1}$, X.B. Bao ${ }^{1}$, W.D. Liu ${ }^{1}$ \\ and C.B. $\mathrm{He}^{1}$ \\ ${ }^{1}$ Key Laboratory of Marine Fishery Molecular Biology of Liaoning Province, \\ Liaoning Ocean and Fisheries Science Research Institute, Dalian, China \\ ${ }^{2}$ College of Marine Life Sciences, Ocean University of China, Qingdao, China \\ Corresponding author: C.B. He \\ E-mail: hechongbo@hotmail.com
}

Genet. Mol. Res. 13 (4): 10727-10740 (2014)

Received November 4, 2013

Accepted February 28, 2014

Published December 18, 2014

DOI http://dx.doi.org/10.4238/2014.December.18.14

\begin{abstract}
Chitinase-like proteins (CLP) are important members of the glycoside hydrolase family 18 (GH18) and are involved in growth control and remodeling processes. In this study, a CLP transcript was isolated and sequenced from the Japanese scallop (Mizuhopecten yessoensis) after screening expressed sequence tags. The full-length complementary DNA of $M$. yessoensis CLP (My-Clp1) was 1555 bp in length, consisting of a 75-bp 5'-untranslated region (UTR), a 160-bp 3'UTR, and a 1320-bp open reading frame bearing characteristics of the GH18 family. The My-Clp1 protein was well conserved, with similar domain structures and architecture across species (e.g., from mollusks to mammals). Expression analysis in healthy tissues and across developmental stages revealed a strong preference for expression; $\mathrm{My}$-Clpl was abundantly expressed in the mantle and throughout metamorphosis, which suggests the involvement of $M y-C l p l$ in the synthesis of extracellular components, and tissue degeneration and
\end{abstract}


remodeling. $M y-C l p 1$ expression was induced after infection with a bacterial pathogen, Vibrio anguillarum, suggesting its involvement in immunity against this intracellular pathogen.

Key words: Japanese scallop Mizuhopecten yessoensis; Chitinase-like protein; mRNA expression; Tissue degeneration

\section{INTRODUCTION}

Chitin, composed of $\beta(1,4)$-linked N-acetyl-D-glucosamine residues, is a naturally occurring polysaccharide found in many life forms, including the exoskeleton and gut linings of insects, cell walls of microorganisms, microfilarial sheaths of parasitic nematodes, and shells of crustaceans (Fuhrman and Piessens, 1985; Kneipp et al., 1998; Vogan et al., 2002; Wills-Karp and Karp, 2004; Lee et al., 2008). The glycoside hydrolase family 18 (GH18), characterized by the ability to hydrolyze chitin enzymatically, has been identified in organisms that synthesize or metabolize chitin, such as insects, yeasts, and crustaceans, and those that do not synthesize or metabolize chitin, such as higher plants and mammals. The GH18 family consists of a specific arrangement of 8 centrally located parallel $\beta$-strands and 8 surrounding $\alpha$-helices (Badariotti et al., 2007). The GH18 family members aid in the protection of organisms from harsh conditions and host anti-parasite/pathogen invasions because they are often constitutively expressed in macrophages and epithelial cells, which provide the first line of defense against exogenous agents, including chitin-containing pathogens (Homer et al., 2006; Mizoguchi, 2006). In the GH18 family, recent studies have identified chitinases and a number of chitinase-like proteins (CLPs), from which chitin could not be catalyzed because of the deletion of a critical amino acid in the catalytic center (Ober and Chupp, 2009).

Increasing numbers of CLPs have been identified from many species in recent years, and they have been implicated in a variety of biological functions. In plants, CLPs function in nodulation and the developmental signaling pathway, which regulates cell proliferation/differentiation and endogenous cytokinin levels (Goormachtig et al., 2001; Lee et al., 2003). Imaginal disc growth factors (IDGFs), the CLPs in Drosophila, are the first polypeptide growth factors to be reported from invertebrates (Kawamura et al., 1999). The IDGF proteins are secreted and transported to target tissues via the hemolymph; they act in conjunction with insulin to stimulate the proliferation, polarization, and motility of imaginal disc cells (Arakane and Kawamura et al., 1999; Muthukrishnan, 2010). In mammals, YKL-40 [a CLP also referred to as a chitinase 3-like-1 and human cartilage glycoprotein-39 (HC-gp39)] was first identified in mouse breast cancer cells and named BRP-39 (Bleau et al., 1999). YKL-40, which has been significantly correlated with the severity of asthma, plays an important role in the homeostasis of several organs in humans, where it regulates tissue remodeling as well as cell proliferation and survival in response to pathological conditions (Giannetti et al., 2004; Kelleher et al., 2005; Recklies et al., 2005; Johansen, 2006). In mollusks, 3 CLPs (Cg-Clp1, Cg-Clp2, and Cg-Clp3) have been identified from the oyster Crassostrea gigas (Badariotti et al., 2006, 2007, 2011). Cg-Clp1 was found to be involved in growth control and remodeling processes in a manner similar to its YKL-40 mammalian counterpart, and $\mathrm{Cg}$-Clp3 plays a role in embryonic development, adult oyster growth, and tissue remodeling during metamorphosis and gonadal restructuring (Badariotti et al., 2006, 2011).

The Japanese scallop (Mizuhopecten yessoensis), widely distributed in the cold seas 
along the coasts of the northern islands of Japan, was introduced to China over 3 decades ago (Nagashima et al., 2005; Li et al., 2007; Liu et al., 2010; He et al., 2012). The annual production of the Japanese scallop had reached 300,000 t in China by 2012 (Han et al., 2012). However, with the rapid expansion and intensification of scallop cultures, pathogen spread, and disease outbreaks have caused massive financial losses to farmers. Thus, additional information on and a greater understanding of the immune system of scallops are necessary. To further clarify the roles of CLPs in the development and immune response of the Japanese scallop, the following were conducted in the present study: 1) cloning the full-length complementary DNA (cDNA) of the $C L P$ gene from the Japanese scallop ( $M y-C l p 1) ; 2$ ) examining the expression of $C L P$ in various tissues; 3 ) examining the expression of $C L P$ at different developmental stages; and 4) evaluating $C L P$ expression in the Japanese scallop challenged with Vibrio anguillarum.

\section{MATERIAL AND METHODS}

\section{Tissue sampling and RNA extraction}

All experimental Japanese scallops (averaging $\sim 80 \mathrm{~mm}$ in shell length for adults) were randomly collected from Dalian Bilong Co., Ltd., China. To determine gene expression in various healthy scallop tissues, samples of 6 tissues, including the mantle, hepatopancreas, gill, kidney, hemocyte, and muscle, of 10 healthy individuals were collected. To determine gene expression during different developmental stages of the scallop, samples from 10 stages, including zygote, 2-cell, blastula, gastrula, trochophore, D, post-D, umbo, juvenile, and metamorphosis, of healthy individuals were collected. All of the above samples were pooled, flashfrozen in liquid nitrogen, and maintained in a $-80^{\circ} \mathrm{C}$ ultra-low freezer for RNA preparation. Total RNA was isolated from different samples using the RNAprep pure Tissue Kit (Tiangen Biotech, China). The quality of RNA was detected by $1 \%$ agarose gel electrophoresis, and the concentration and purity were determined spectrophotometrically by measuring their absorbance at 260 and $280 \mathrm{~nm}$ using the Implen Nanophotometer (Implen, Germany) (Li et al., 2012). First-strand cDNA was synthesized using the PrimeScript ${ }^{\mathrm{TM}}$ First-Strand cDNA Synthesis Kit (TaKaRa, Japan).

\section{Identification and sequencing of $M y-C l p 1$ cDNA}

The Japanese scallop mantle cDNA library that we constructed was previously used as the EST database (Li et al., 2010). According to BLASTX, the clone MYMAN10A12 (GenBank ID: GR867827) is homologous to a partial CLP sequence. Rapid amplification of cDNA ends (RACE) was performed on a partial CLP sequence to obtain full-length cDNA. RACE was performed using the SMART RACE cDNA Amplification Kit (Clontech/BD Biosciences, Japan). The final polymerase chain reaction (PCR) product was cloned into pMD19-T Simple Vectors $(\mathrm{TaKaRa})$ and sequenced by the $\mathrm{AB} 3500$ genetic analyzer.

\section{In silico characterization of $M y-C l p 1$}

To fully characterize $\mathrm{My}$-Clp1, several molecular models were constructed, including the architecture of conserved domains, homology modeling of catalytic domains, a phylogenetic tree, and a multiple-sequence alignment. The predicted amino acid sequences of 
the $M y$-Clpl gene were assessed via BLAST (http://www.ncbi.nlm.nih.gov/blast/Blast.cgi). Conserved domain structures were determined using the SMART domain program and the Conserved Domain Database (CDD) of NCBI (Letunic et al., 2006). The presumed tertiary structures of $M y$-Clpl were established using the SWISS-MODEL prediction algorithm (http://swissmodel.expasy.org/) (Arnold et al., 2006; Kiefer et al., 2009). A phylogenetic tree of selected $C L P \mathrm{~s}$ was constructed using the neighbor-joining method via MEGA 4.0 (Tamura et al., 2007). The bootstrap trials were replicated 1000 times to derive the confidence values for the phylogeny analysis. The ClustalW Multiple Alignment program was used for multiple alignments of MSTN genes (Thompson et al., 2002). Conserved features from this alignment were labeled accordingly. The presence and location of the signal peptide was predicted by SignalP 3.0 (Bendtsen et al., 2004). The molecular mass (Mw) and theoretical isoelectric point (pI) of the protein was predicted using a Compute $\mathrm{pI} / \mathrm{Mw}$ tool (http://web.expasy.org/compute_pi/).

\section{Bacterial challenge and sample collection}

Prior to feeding, adult scallops were acclimated to the laboratory condition for 1 week. Scallops were divided into control and experimental groups for the bacterial challenge experiment. They were maintained at a temperature of approximately $15^{\circ} \mathrm{C}$ under a natural photoperiod (12-h light/12-h dark) in rectangular plastic tanks $\left(10 \mathrm{~m}^{3}\right)$ containing filtered, continuously aerated seawater (28-30\% salinity). Scallops receiving an injection of $50 \mu \mathrm{L}$ live

$V$. anguillarum $\left(3 \times 10^{7} \mathrm{CFU} / \mathrm{mL}\right)$ in saline suspension were used as the challenge group, while animals in the control group were injected with the same amount of sterile seawater. Scallops were returned to the seawater tanks, and the hemolymphs of 3 individuals from each tank were randomly sampled from the challenged and control groups at $0,3,6,12,24$, and $36 \mathrm{~h}$ post-injection. Hemolymph samples were immediately centrifuged for $10 \mathrm{~min}(1000 \mathrm{~g}$, $4^{\circ} \mathrm{C}$ ), frozen in liquid nitrogen, and stored at $-80^{\circ} \mathrm{C}$ until RNA extraction and analysis.

\section{Gene expression analysis of $\mathrm{My}-\mathrm{Clp} 1$}

The levels of $M y$-Clp1 mRNA expression in different tissues throughout various developmental stages and hemocytes following bacterial stimulation were detected by real-time quantitative reverse transcription-PCR (qRT-PCR) in Mx3005 $\mathrm{p}^{\mathrm{TM}}$ real-time thermal cycler with the PrimeScript ${ }^{\mathrm{TM}}$ RT Reagent Kit (TaKaRa). The primers $\mathrm{Q} \beta$-actin-F and $\mathrm{Q} \beta$-actin-R (GenBank accession No. GU596498) were used to amplify part of the M. yessoensis $\beta$-actin coding sequence as an internal control to calibrate the cDNA template for corresponding samples. The reaction mixture was comprised of $10 \mu \mathrm{L} 2 \mathrm{X}$ SYBR $^{\circledR}$ Premix Ex Taq ${ }^{\mathrm{TM}}, 0.8 \mu \mathrm{M}$ of each primer (i.e., forward and reverse), $1 \mu \mathrm{L}$ cDNA template, and then filled to a final volume of $20 \mu \mathrm{L}$ with distilled water. The amplification conditions were as follows: denaturation and enzyme activation at $95^{\circ} \mathrm{C}$ for $30 \mathrm{~s}$, followed by 40 cycles of $94^{\circ} \mathrm{C}$ for $5 \mathrm{~s}, 55^{\circ} \mathrm{C}$ for $20 \mathrm{~s}$, and $72^{\circ} \mathrm{C}$ for $20 \mathrm{~s}$. Each sample was run in triplicate along with the internal control gene. A dissolution curve analysis of the amplification products was performed to confirm the specificity of the qRT-PCR products. The CT method $\left(2-{ }^{\Delta \Delta \mathrm{Ct}}\right)$ was used to calculate relative changes in My-Clp1 mRNA expression (Arocho et al., 2006). 


\section{Data analysis}

Statistical analysis was performed using the SPSS 13.0 software least significant difference (LSD) $t$-test. All data are reported as means $\pm \mathrm{SD} / \mathrm{SE}$ for $\mathrm{N}=10$ animals per group for three independent experiments. One-way ANOVA followed by the LSD test was used to determine significant differences in the mean values among the different groups.

\section{RESULTS}

\section{Cloning and sequencing of $M y-C l p 1$ cDNA}

A 1555-bp nucleotide sequence representing the full-length cDNA sequence of My-Clpl was obtained and deposited into GenBank (accession No. KC876641). The My-Clp1 cDNA contained an open reading frame of $1320 \mathrm{bp}$ encoding 439 amino acids with a 75-bp 5'-untranslated region (UTR) and a 160-bp 3'-UTR (Figure 1). The mature protein had a theoretical $\mathrm{pI}$ of 6.37 and a predicted $\mathrm{Mw}$ of $50.4 \mathrm{kDa}$. A typical polyadenylation signal sequence (AATAAA) existed $13 \mathrm{bp}$ upstream of the poly (A) sequences. The N-terminus of the polypeptide was composed of a signal peptide of 22-amino acid residues.

\section{Homology analysis of $M y-C l p 1$}

Multiple-sequence alignment revealed the significant identities of the $\mathrm{My}$-Clpl amino acid sequence with those of previously identified GH18 family members, especially in the Glyco_18 domain (Figure 2). The predicted amino acid sequence of $M y$-Clpl was similar to that of the CLPs in Homo sapiens (34\% positive), Drosophila melanogaster (32\%), C. gigas (49\%), and Pinctada margaritifera (43\%). The amino acid C-terminal sequence contained 55 more amino acids than $\mathrm{Hs}_{s}$-HC-gp39 and $\mathrm{Dm}$-Cht9, and 34 less than $\mathrm{Cg}$-Clp and $\mathrm{Pm}$-Clp, with unknown functions. According to the homology model constructed by the SWISS-MODEL program, $M y-C l p 1$ had a similar structure to that of other CLPs and exhibited the $(\beta / \alpha)_{8}$ TIM barrel structure (Figure 3).

\section{Phylogenetic analysis of $\mathrm{My}$-Clp1}

In order to analyze the $M y$-Clpl gene in the larger genomic context, phylogenetic analysis was conducted based on amino acid sequences encoded by 18 CLP genes (Figure 4). The CLP homologous proteins were separated into 3 distinct groups, including Lophotrochozoa, Deuterostoma, and Ecdysozoa. In all cases, the $M y-C l p 1$ fell within the clades that also included mollusk CLPs, thus confirming their identities as suggested by similarity comparisons. Analyses using several algorithms, such as neighbor joining and maximum parsimony methods, yielded similar phylogenetic trees. While the evolutionary relationships observed among the CLPs appear to be consistent, further studies on other CLPs would assist in the construction of a more comprehensive phylogeny. 
1 cgetcgtgtgtggatgtctgtttagecttttagcgatatcaaaaaaggactttctggata

61 catcaaatcatcaagATGGACGCGCAAGCCGTTCTTCGAAGTGTACTTGTCCTCCTGTTA

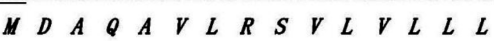

121 CACGTCATCCTCACAGCAGCCCAGTTCAAACGGGTGTGTTACTACAGTGGTTGGTCACTG

$\begin{array}{lllllllllllllllllllll}16 & \boldsymbol{H} & \boldsymbol{V} & \boldsymbol{I} & \boldsymbol{L} & \boldsymbol{T} & \boldsymbol{A} & \boldsymbol{A} \downarrow \downarrow & \mathrm{Q} & \mathrm{F} & \mathrm{K} & \mathrm{R} & \mathrm{V} & \mathrm{C} & \mathrm{Y} & \mathrm{Y} & \mathrm{S} & \mathrm{G} & \mathrm{W} & \mathrm{S} & \mathrm{L}\end{array}$

181 TACCGTGACAAAGAGAGGGGTTTGGCTCCCGAAGACATCGATCCCTATCTCTGTACCCAT

$\begin{array}{lllllllllllllllllllllllllllllllllll}36 & \text { Y } & \text { R } & \text { D } & \text { K } & \text { E } & \text { R } & \text { G } & \text { L } & \text { A } & \text { P } & \text { E } & \text { D } & \text { I } & \text { D } & \text { P } & \text { Y } & \text { L } & \text { C } & \text { T } & \text { H }\end{array}$

241 ATTGTCTATGCCTATGCTACCCTTGATGATACGGGAACTCGGATCATTGTACCGGACGGA

$\begin{array}{lllllllllllllllllllll}56 & \text { I } & \text { V } & \text { Y } & \text { A } & \text { Y } & \text { A } & \text { T } & \text { L } & \text { D } & \text { D } & \text { T } & \text { G } & \text { T } & \text { R } & \text { I } & \text { I } & \text { V } & \text { P } & \text { D } & \text { G }\end{array}$

301 TATAAAGCAGAGGAACTCAACTTGTTCCGTAGGTTCCATAGCATGCGCGCCAAGAACGAT

$\begin{array}{llllllllllllllllllllll}76 & \mathrm{Y} & \mathrm{K} & \mathrm{A} & \mathrm{E} & \mathrm{E} & \mathrm{L} & \mathrm{N} & \mathrm{L} & \mathrm{F} & \mathrm{R} & \mathrm{R} & \mathrm{F} & \mathrm{H} & \mathrm{S} & \mathrm{M} & \mathrm{R} & \mathrm{A} & \mathrm{K} & \mathrm{N} & \mathrm{D}\end{array}$

361 GATCTTGTGATGATGTTGTCAATTGGAGGCTGGGCTACCGACAGTAAGCTTTTTTCTAAG

$\begin{array}{llllllllllllllllllllll}96 & \text { D } & \text { L } & \text { V } & \text { M } & \text { M } & \text { L } & \text { S } & \text { I } & \text { G } & \text { G } & \text { W } & \text { A } & \text { T } & \text { D } & \text { S } & \text { K } & \text { L } & \text { F } & \text { S } & \text { K }\end{array}$

421 ACAGTTTCTTCGCAAGAAAACATGCAGATCTTCGCGGGGGAAGCAATCAACTACCTCCGC

$\begin{array}{llllllllllllllllllllll}116 & \mathrm{~T} & \mathrm{~V} & \mathrm{~S} & \mathrm{~S} & \mathrm{Q} & \mathrm{E} & \mathrm{N} & \mathrm{M} & \mathrm{Q} & \mathrm{I} & \mathrm{F} & \mathrm{A} & \mathrm{G} & \mathrm{E} & \mathrm{A} & \mathrm{I} & \mathrm{N} & \mathrm{Y} & \mathrm{L} & \mathrm{R}\end{array}$

481 AAACACGACTTCGACGGACTGGACATCGACTGGCAGTTCCCCGCCACGCGAGGCAGTCCG

$\begin{array}{lllllllllllllllllllll}136 & K & H & D & F & D & G & \text { L } & \text { D } & \text { I } & \text { D } & \text { W } & \text { Q } & \text { F } & \text { P } & \text { A } & \text { T } & R & G & S & P\end{array}$

541 CCAGAGGACGTAGAGCGCTACTACAGATTTTTGAGGCTGGTCCAATGGGAGTTTGAGCAC

$\begin{array}{llllllllllllllllllllll}156 & P & E & D & V & E & R & Y & Y & R & F & L & R & L & V & Q & W & E & F & E & H\end{array}$

601 GAGGAGGAGCCTGACGATAAGAGTACCCTGATCCTGACTATAGCCGTAGACCCGACAGTG

$\begin{array}{llllllllllllllllllllll}176 & E & E & E & P & D & D & K & S & T & L & I & L & T & I & A & V & D & P & T & V\end{array}$

661 GAGAGGGCTTCCATCTCATACGACCTCCCCCGATATTCCAGGTGGGTGAACTGGATAAAC

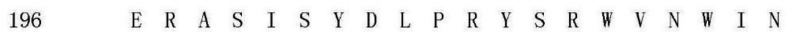

721 ATGAAGATGTTTGACTTCTACGGCCACTGGGATGACCCGACTGTTGCCAATCACCACAGC

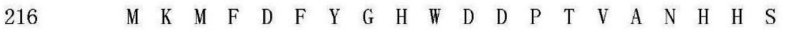

781 GCCCTATACAGTGCCAAGGATCCCAAAAATGTGAACAATCTTAGTCGATACTGGGTAAAC

$\begin{array}{llllllllllllllllllllll}236 & \text { A } & \text { L } & \text { Y } & \text { S } & \text { A } & \text { K } & \text { D } & \text { P } & \text { K } & \text { N } & \text { V } & \text { N } & \text { N } & \text { L } & \text { S } & R & \text { Y } & \text { W } & \text { V } & \text { N }\end{array}$

841 AAGGGCGTACCTCGTCACAAGATCGTACTAGGACTGCCCATGTACGGCCGCTCATTTTCA

$\begin{array}{llllllllllllllllllllll}256 & K & G & V & P & R & H & K & \text { I } & \text { V } & \text { L } & G & \text { L } & \text { P } & \text { M } & \text { Y } & G & R & S & F & S\end{array}$

901 CTGGCCAACACCAACTACACACAGCCCGGGGCCCCCGCCCTCGGTCCGGGATCTGATGAA

$\begin{array}{llllllllllllllllllllll}276 & \mathrm{~L} & \text { A } & \text { N } & \text { T } & \text { N } & \text { Y } & \text { T } & \text { Q } & \text { P } & \text { G } & \text { A } & \text { P } & \text { A } & \text { L } & G & \text { P } & \text { G } & \text { S } & \text { D } & \text { E }\end{array}$

961 GGCGACGGCTATCCCATAGCACAGCTTTGTCATCTGATTAAAAATGGTGCCAGGGAAATG

$\begin{array}{llllllllllllllllllllll}296 & G & D & G & \text { Y } & \text { P } & \text { I } & \text { A } & \text { Q } & \text { L } & \text { C } & \text { H } & \text { L } & \text { I } & \text { K } & \text { N } & \text { G } & \text { A } & \text { R } & \text { E } & \text { M }\end{array}$

1021 CTGATCGCAGATAAACGAGTGCCTTATGTGGTCATTGGCGATGAGTGGATCGGGTATGAC

$\begin{array}{llllllllllllllllllllll}316 & \text { L } & \text { I } & \text { A } & \text { D } & \text { K } & \text { R } & \text { V } & \text { P } & \text { Y } & \text { V } & \text { V } & \text { I } & \text { G } & \text { D } & \text { E } & \text { W } & \text { I } & \text { G } & \text { Y } & \text { D }\end{array}$

1081 AATCCCGAGAGTATCAAACAAAAGGCTCGAATCGCATTCAACAACTTCCTGGGTGGCGTC

$\begin{array}{llllllllllllllllllllll}336 & \mathrm{~N} & \mathrm{P} & \mathrm{E} & \mathrm{S} & \mathrm{I} & \mathrm{K} & \mathrm{Q} & \mathrm{K} & \mathrm{A} & \mathrm{R} & \mathrm{I} & \mathrm{A} & \mathrm{F} & \mathrm{N} & \mathrm{N} & \mathrm{F} & \mathrm{L} & \mathrm{G} & \mathrm{G} & \mathrm{V}\end{array}$

1141 ATGATCTGGACGTTGGACATGGATGACCACCGTGGCGCATGTGGTCGGCCCTACCCGTTG

$\begin{array}{llllllllllllllllllllll}356 & \text { M } & \text { I } & \text { W } & \text { T } & \text { L } & \text { D } & \text { M } & \text { D } & \text { D } & \text { H } & R & G & \text { A } & C & G & R & P & Y & P & L\end{array}$

1201 ATAAACGCCGCGTTGGACGGTCTCAACGCCCGCCACGGGTATAAAGAGCTCATCACAAAC

$\begin{array}{lllllllllllllllllllll}376 & \text { I } & N & A & A & L & D & G & L & N & A & R & H & G & Y & K & E & L & \text { I } & T & N\end{array}$

1261 GCCCTGCAGGACCAAGCTCAACAGGCCGCCGCTAAGAAGGAAATATATAGACAGAGAGCG

$\begin{array}{llllllllllllllllllllll}396 & \text { A } & \text { L } & \text { Q } & \text { D } & \text { Q } & \text { A } & \text { Q } & \text { Q } & \text { A } & \text { A } & \text { A } & \text { K } & \text { K } & \text { E } & \text { I } & Y & R & \text { Q } & \text { R } & \text { A }\end{array}$

1321 CTGGGATGGGAAATGCAGGACAAACAAGAACAGATTCATAACCAACAAAGAGGAGGTCGT

$\begin{array}{llllllllllllllllllllll}416 & \mathrm{~L} & G & \text { W } & E & M & Q & D & K & Q & E & Q & \text { I } & H & N & Q & Q & R & G & G & R\end{array}$

1381 AGACGAGGCTGGTGAgctgtaactcagagttacgactactggttggccagcacatggtgt

$436 \quad$ R $\quad R \quad G \quad W \quad *$

1441 tatggtgtaatttaaataaatgcattaactctaaagataccgetattctccggtgtagtt

1501 acttcaatttcccaaaaaataaatagaatatttataaaaaaaaaaaaaaaa

Figure 1. Nucleotide and deduced amino acid sequences of $M y$-Clp1 cDNA. The coordinates of the cDNA and amino acids are indicated in the left margin. 5'- and 3'-untranslated regions are indicated using lowercase letters. The translation start codon is ATG, the termination codon is TGA, and the polyadenylation signal sequences AATAAA are shown in bold font and underlined. The termination is labeled by an asterisk. The signal peptide is italicized, and the downward-pointing arrow (A22 $\downarrow$ Q23) indicates the probable cleavage site for its removal. 


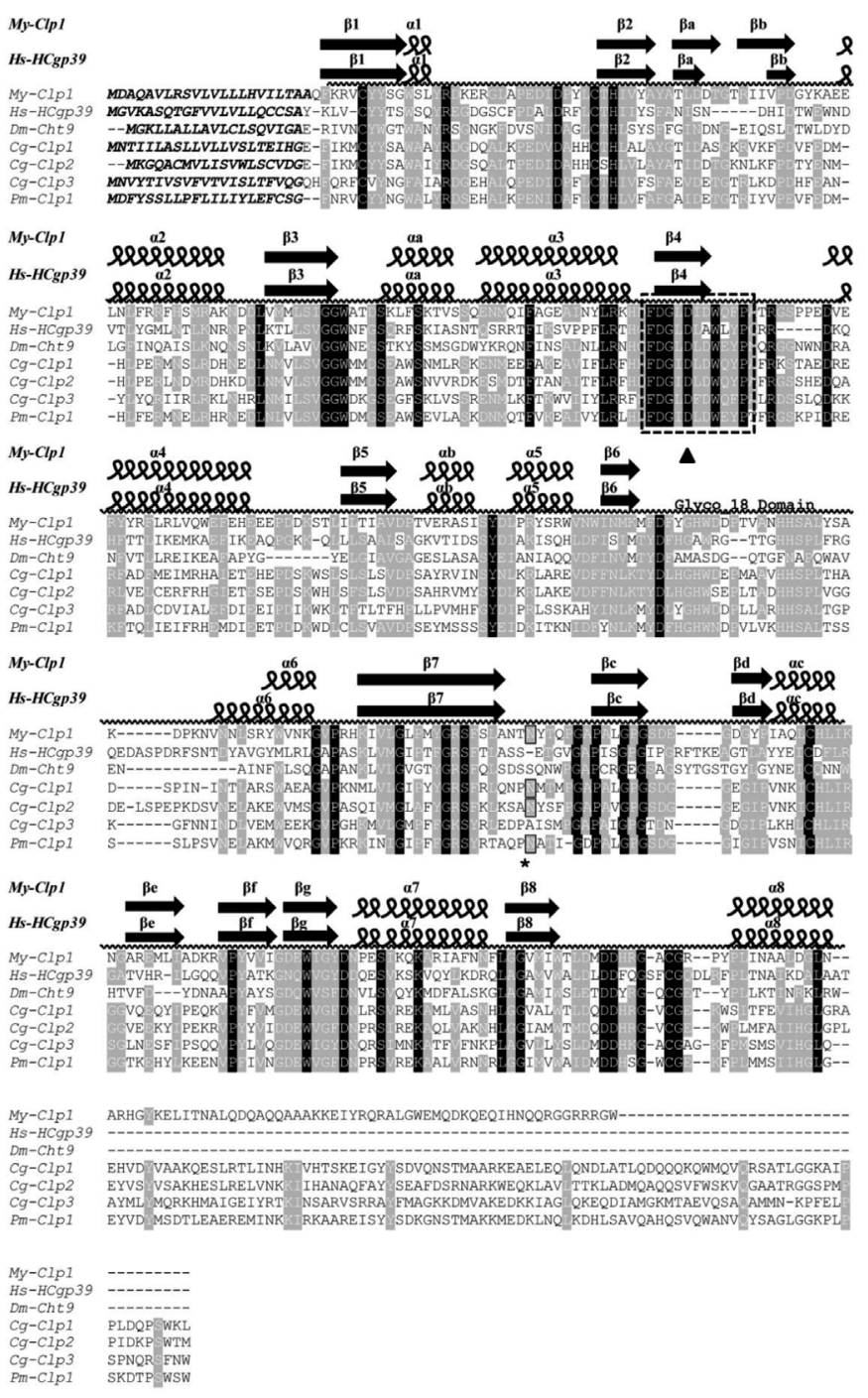

Figure 2. Multiple-sequence alignment of the deduced amino acid sequences of $M y$-Clpl with CLPs of the GH18 family. The predicted amino acid sequence of $M y$-Clp1 is aligned with the amino acid sequence of 6 CLPs from Homo sapiens (Hs-HC-gp39), Drosophila melanogaster (Dm-Cht9), Crassostrea gigas (Cg-Clp1, Cg-Clp2, and $\mathrm{Cg}$-Clp3), and Pinctada margaritifera (Pm-Clp1). Conserved residues are shaded in gray (identical) or black (identical in all of the proteins). Amino acids of the predicted signal peptide are shown in bold italic letters. Dashes indicate gaps in the amino acid sequence when compared to the other sequences. The wave line above the sequence alignment delimits the Glyco_18 domain. The broken black box marks the extent of the active center. Head arrow indicates the position of residue required for catalytic activity in bacterial chitinase (Watanabe et al., 1993). Black box and asterisk indicate the potential site for $N$-glycosylation $(\mathrm{NXT} / \mathrm{S})$. The secondary structure elements of $M y$-Clp1 and $H s$-HC-gp39 are depicted in black above the alignment. Squiggles represent helices, and arrows represent $\beta$ strands. Helices and $\beta$ strands that are part of the $(\alpha \beta)_{8}$ barrel are numbered, and secondary elements outside the barrel are labeled with letters. $C g$ - $C l p 1$ secondary structure elements are predicted using the SWISSMODEL software (Badariotti et al., 2006). The GenBank accession Nos. of the genes are as follows: Hs-HC-gp39, NP001267; $\mathrm{Dm}$-Cht9, NP611543; $\mathrm{Cg}$-Clp1, CAI96029; $\mathrm{Cg}$-Clp2, CAI96023; $\mathrm{Cg}$-Clp3, CAI96024; and Pm-Clp1, H2A0L4.1 (UniProtKB/Swiss-Prot). 


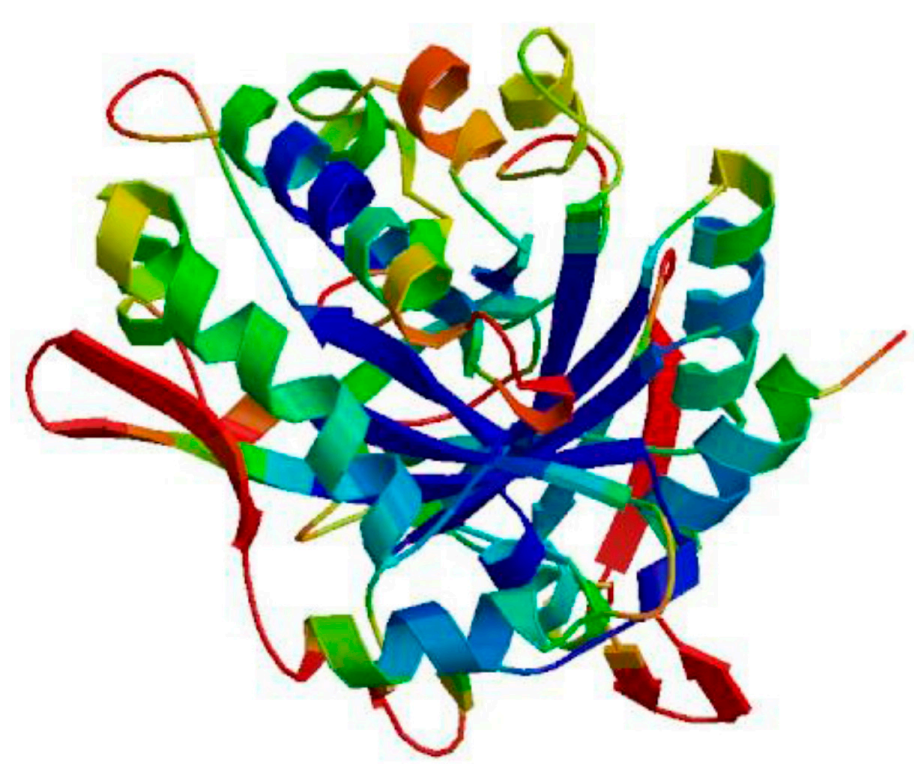

Figure 3. Homology modeling of $M y$-Clpl catalytic domain. The SWISS-MODEL program was used to generate the models. Human HC-gp39 was used as a template.

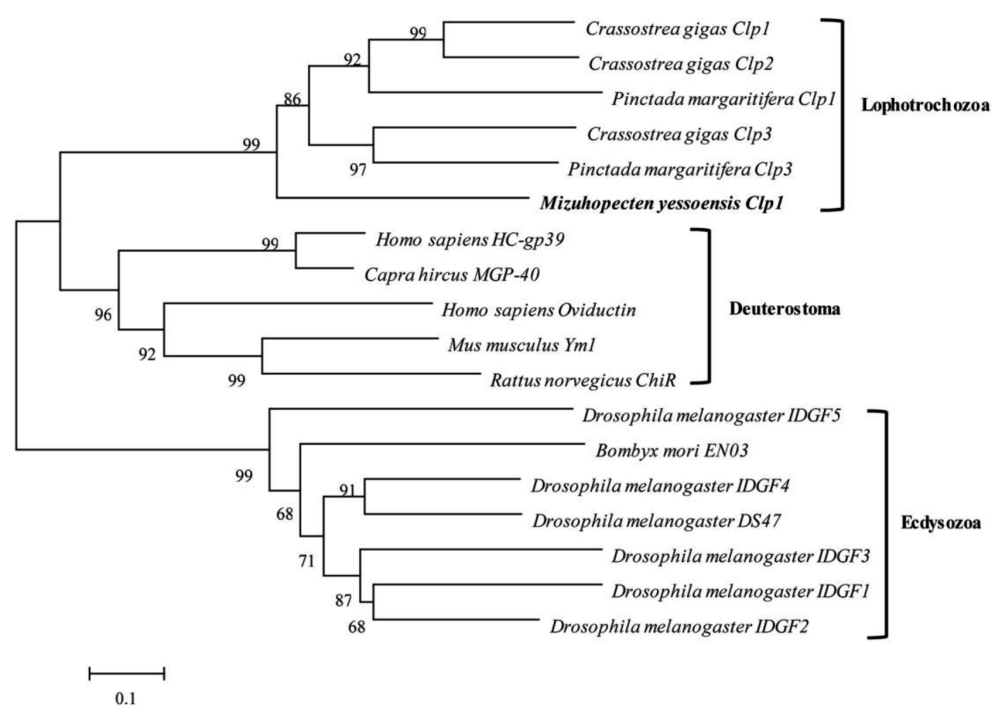

Figure 4. Phylogenetic analysis of chitinase-like proteins from Mizuhopecten yessoensis and other species. A phylogenetic tree was constructed by the neighbor-joining method based on the protein sequences of 18 chitinaselike proteins. Sequences used for the analysis were as follows: Crassostrea gigas Clp1, CAI96029; C. gigas Clp2, CAI96023; C. gigas Clp3, CAI96024; Pinctada margaritifera Clp1, H2A0L4.1; P. margaritifera Clp 2, P86955.1; Homo sapiens HC-gp39, NP_001267; Capra hircus MGP-40, AAL87007; H. sapiens Oviductin, Q12889; Mus musculus Ym1, NP 034022; Rattus norvegicus ChiR, XP 227568; Drosophila melanogaster IDGF1, Q8MX41; D. melanogaster IDGF̄2, NP_477257; D. melanogaster IDḠF3, NP_477256; D. melanogaster IDGF4, NP_511101; D. melanogaster IDGF5, Q870R7; D. melanogaster DS47, NP_4777081; and Bombyx mori EN03, BAB16 695. 


\section{Expression of $M y$-Clp1 in developing and adult tissues}

Real-time qRT-PCR was used to determine the tissue distribution and temporal pattern of $M y$-Clp1 expression during the developmental stages of M. yessoensis. My-Clp1 was expressed in the tissues tested, including the mantle, hepatopancreas, gill, kidney, and hemocyte, with the exception of the muscle tissue (Figure 5). The expression level of MyClpl detected in the mantle, which was the highest among all the tissues tested, was almost 27-fold greater than that in the hepatopancreas, which yielded the second highest expression level. During the developmental stages, $\mathrm{My}$-Clpl transcripts were expressed at moderate levels in all embryonic and larval stages, including zygote, 2 cells, blastula, gastrula, trochophore, D, post-D, umbo, and juvenile (Figure 6). However, the greatest transcriptional abundance of $M y$-Clpl was observed during metamorphosis, which was 15-170-fold greater than those in the other stages.

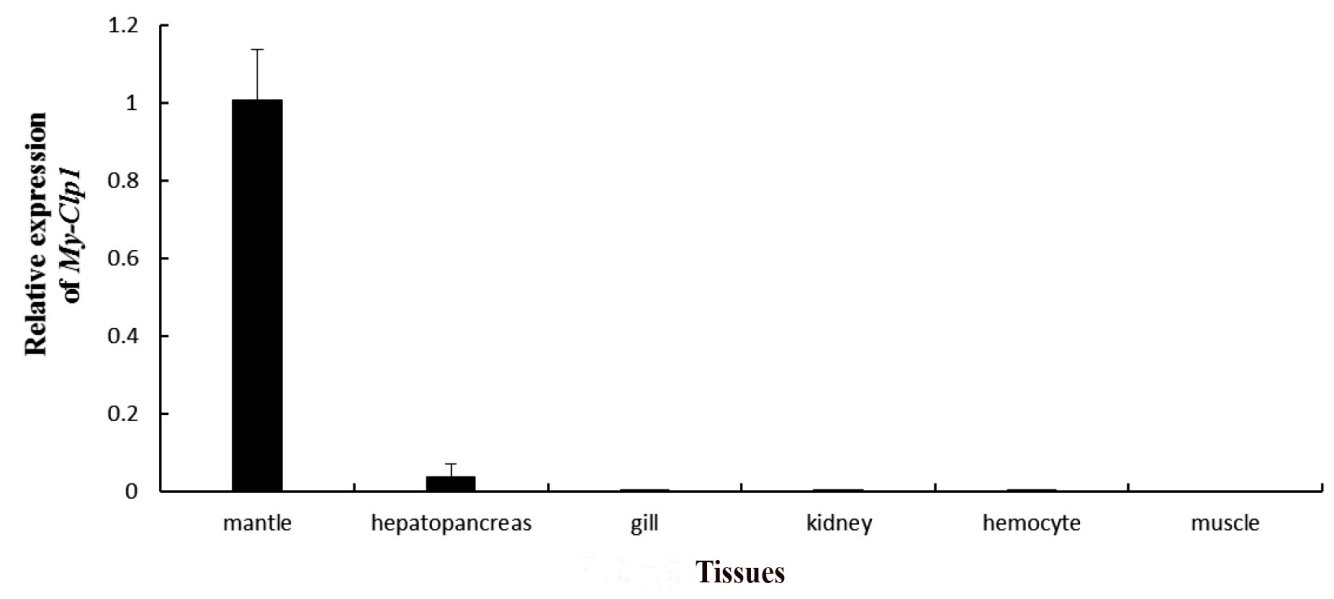

Figure 5. Tissue distribution of the $M y-C l p 1$ transcript. The value of relative expression was achieved by comparing the expression level of CLP with that of $\beta$-actin. Each symbol and vertical bar represent means \pm standard deviation.

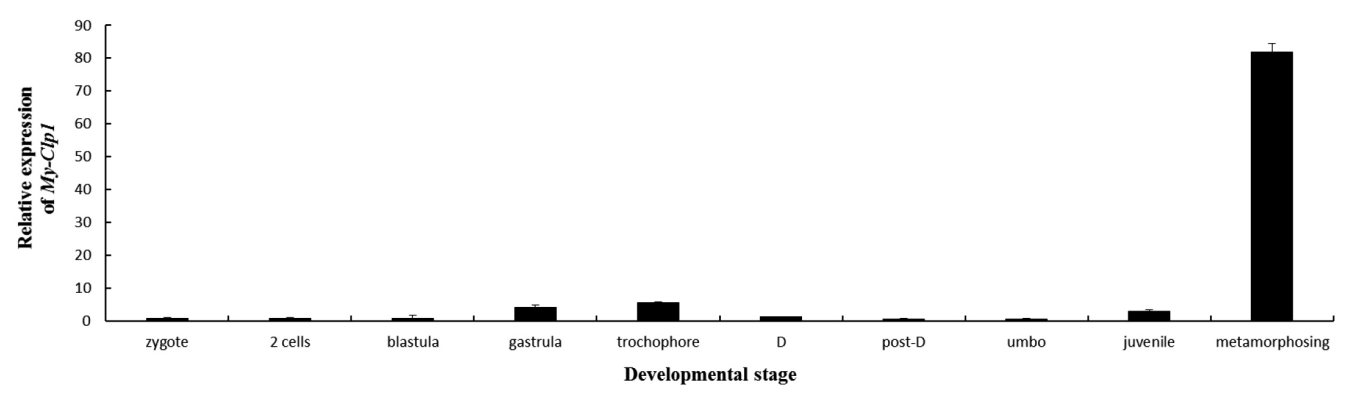

Figure 6. Expression of $M y-C l p 1$ during different developmental stages. The value of relative expression was achieved by comparing the expression level of CLP with that of $\beta$-actin. Each symbol and vertical bar represent means \pm standard deviation. 


\section{Expression of $M y$-Clp1 after bacterial challenge}

In order to understand the role of $M y$-Clpl in response to disease infection with $V$. anguillarum, qRT-PCR analysis was conducted to determine the expression patterns of My-Clp1 in an infected hemolymph. As shown in Figure 7, a marked increase in $\mathrm{My}$-Clp1 expression was observed at 12 and $24 \mathrm{~h}$, with the highest level occurring at $12 \mathrm{~h}$ (11.6-fold increase, $\mathrm{P}<0.05$ ). However, the expression level of $M y$-Clp1 in the challenge group began to decrease after $12 \mathrm{~h}$ and dropped below the initial level of expression at $36 \mathrm{~h}$, which was 15 -fold less than that in the control group. There was no significant difference in the expression levels between the challenge and control groups from 0 to $9 \mathrm{~h}$ post injection, except at 6 $\mathrm{h}$ when $\mathrm{My}$-Clpl in the control group showed an unusual expression pattern.

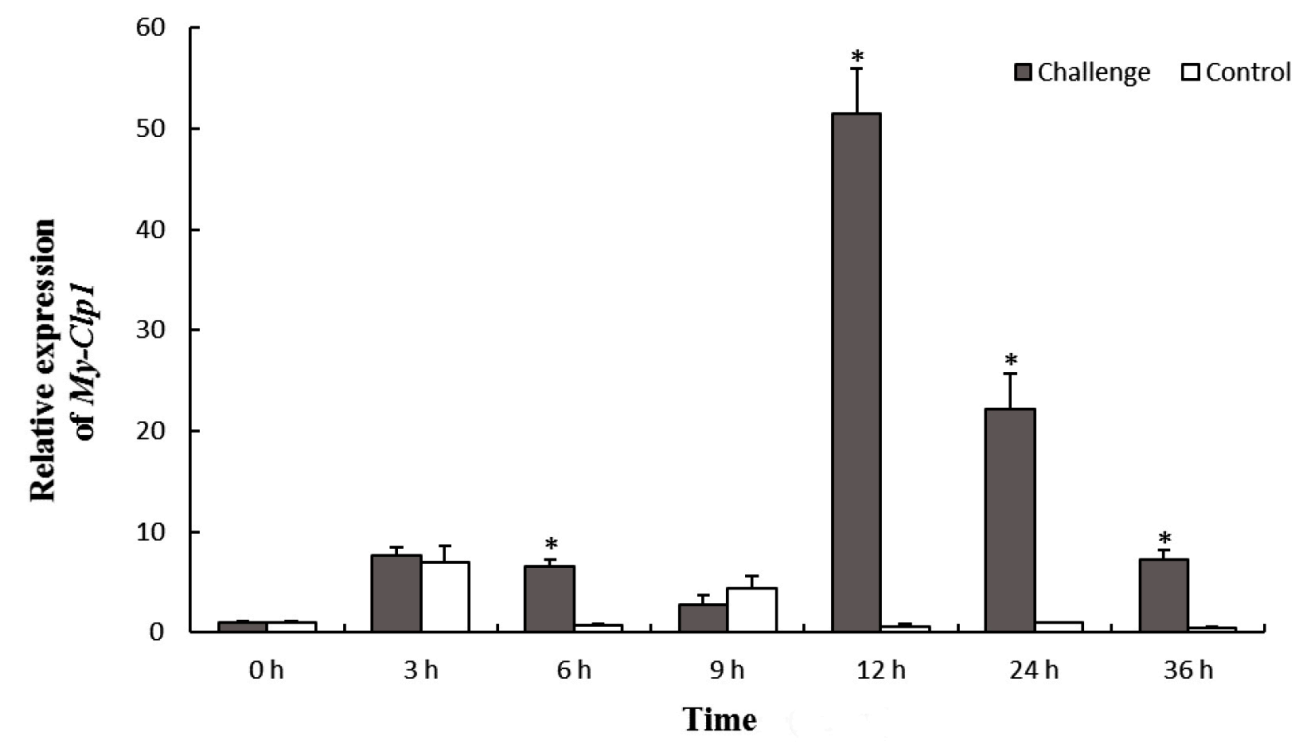

Figure 7. Temporal expression of $M y-C l p l$ in the hemocytes of the Japanese scallop injected with Vibrio anguillarum. The value of relative expression was achieved by comparing the expression level of CLP with that of $\beta$-actin. Each symbol and vertical bar represent means \pm SD. of 3 specimens. Significant differences were indicated with one asterisk at $\mathrm{P}<0.01$.

\section{DISCUSSION}

CLPs are critical for the regulation of cell proliferation, tissue remodeling, and embryonic development. CLP genes have been reported from human, goat, rat, and D. melanogaster individuals (Bleau et al., 1999; Kawamura et al., 1999; Giannetti et al., 2004; Kelleher et al., 2005; Recklies et al., 2005; Johansen, 2006; Arakane and Muthukrishnan, 2010). However, research on the characterization of CLPs has been limited in the Japanese scallop and mollusks, in general. In this study, we conducted sequencing and analyzed a CLP gene from the Japanese scallop. We characterized the gene and protein structure, tissue expression, developmental expression, and expression after bacterial infection.

The CLP homolog identified from M. yessoensis encoded for 439 amino acids. A pre- 
dicted signal peptide was observed in the deduced amino acid sequence of $M y$-Clpl, similar to CLPs in other organisms, which suggests the classification of My-Clp1 as a secreted protein. The full-length cDNA contained a polyadenylation signal (AATAAA) in the 3'-end UTR, which is common among the mRNAs of inflammatory molecules (Shen et al., 2011). The low levels of sequence identity in the C-terminal regions, probably caused by the relatively low selection pressure during evolution, may contribute little to the structure and function of CLPs (Badariotti et al., 2007).

The observations that $\mathrm{My}$-Clpl contained conserved domain structures and architectures, such as the Glyco_18 domains and active centers, provide further evidence for the high degree of sequence conservation of this gene throughout its evolutionary history because all CLPs from other selected species exhibited the same domains based on amino acid sequence homology. The Glyco_18 domains in the homology modeling, containing $~ 370$ amino acids, are highly conserved and generate the $\beta_{8} \alpha_{8}$ barrel structure, which is crucial for the function of these enzymes. High conservation and the principal, basic function of the GH18 family genes are also indicated by multiple-sequence alignment. $M y$-Clpl exhibited abolishing catalytic activity similar to that of the CLPs in C. gigas, wherein the glutamate residue that is critical for chitinase activity is substituted by a glutamine at the location of the active center (Watanabe et al., 1993; Badariotti et al., 2006, 2011). Zhu et al. (2004) also reported that Cht12, Cht13, and Cht14 in Drosophila, lacking the glutamic acid residue that has been identified as the proton donor in the catalytic mechanism, may be involved in cell-to-cell communication or insect immunity. IDGFs, in which the glutamate residue is replaced by glutamine, are believed to have evolved from an ancestral chitinase and possess growth-promoting activities (Kawamura et al., 1999; Zhu et al., 2004).

Phylogenetic analysis suggested that the CLPs in Ecdysozoa, especially IDGFs, have appeared from a recent duplication event based on the grouping of mollusk proteins into the subfamily comprised of mammalian CLPs. This result is supported by the research on C. gigas CLPs, which indicates that Ecdysozoa CLPs converged into their corresponding clade separately (Badariotti et al., 2011). The data also revealed that Lophotrochozoa and Ecdysozoa CLPs underwent more divergence events than those of Deuterostoma. The ancestral origin of $M y$-Clpl is ambiguous according to its position on the phylogenetic tree. Additional CLP sequences from closely related species are needed to more accurately assess the phylogenetic position of $\mathrm{My}$-Clpl.

In order to better understand the biological role of CLP, the expression profiles of $M y-C l p 1$ mRNAs in different tissues and at various developmental stages were investigated by real-time qRT-PCR analysis. $\mathrm{My}$-Clp1 transcripts were detected in almost all tissues tested, with the exception of the muscle tissue. This result is not in agreement with those reported for C. gigas, which indicate a relatively low expression level in the muscle tissue. Furthermore, it was reported that the Chit 2 gene was highly expressed in the muscle tissue (Badariotti et al., 2006, 2007, 2011). This may be due to the lack of expression during specific stages of growth and indicates that the biological activities may be spatially partitioned among the family members. $\mathrm{My}$-Clpl was predominantly expressed in the mantle tissues. As the mantle is responsible for shell formation and body growth via secretion of the shell organic matrix and cell proliferation, $\mathrm{My}$-Clpl was proposed as a key regulator responsible for the synthesis of extracellular components and the proliferation of mantle cells (Badariotti et al., 2007). In addition to the high expression level in the mantle, CLPs in C. gigas were also reported to be highly expressed in the digestive gland, in which proliferation and differentiation of digestive 
stem cells regularly and actively proceeds to counteract the high rate of degeneration of functional digestive cells (Giard et al., 1998; Badariotti et al., 2006).

My-Clp1 transcripts were detected throughout various developmental stages and were predominantly expressed during metamorphosis. Metamorphosis is the ultimate stage of scallop development, which is characterized by the degeneration and remodeling of larval tissues to produce adult tissues (Burke, 1983). In light of the high level of expression of My-Clp1 during metamorphosis, $\mathrm{My}$ - $\mathrm{Clpl}$ was expected to play roles in tissue degeneration and remodeling. Similar results were reported from a study on C. gigas CLPs (Badariotti et al., 2006, 2007, 2011). Clearly, there remains great interest in the investigation of additional $C L P$ genes to determine whether the predominant level of expression during metamorphosis occurs in other mollusks.

The temporal expression pattern of $M y$-Clpl following the $V$. anguillarum challenge was observed to further understand the basic function of $M y-C l p l$ in the immune response. The upregulation of $M y$-Clpl expression after the challenge, especially at 12 and $24 \mathrm{~h}$, suggests that $M y-C l p 1$ is associated with the M. yessoensis innate immune defense mechanism. In addition, it appears that hemocytes were involved in the immune response to the $V$. anguillarum challenge. The low level of sequence divergence, especially for the Glyco_18 domains, observed between M. yessoensis and CLPs from other species indicates the importance of CLPs in pathogen recognition and implies they may recognize the same microbial ligands. For instance, the CLPs in mammals and C. gigas have been shown to act as lectins in the immune response to chitinous pathogens (Houston et al., 2003; Badariotti et al., 2006). The immune response of $\mathrm{My}-\mathrm{Clpl}$ to bacteria may be caused by the combination of CLP and specific components of the cell wall, such as LPS, which harbor GlcNAc, the constituent of chitin in their molecular structure (Badariotti et al., 2007).

In conclusion, a chitinase-like protein gene was successfully isolated and characterized from M. yessoensis. It shares important structural elements with CLPs of other species, which indicates the evolutionary restraints on the mutation of key domains and motifs. $M y-$ $C l p 1$ was abundantly expressed in the mantle and during metamorphosis. The expression level of $M y$-Clpl was significantly induced after infection with $V$. anguillarum. These results suggest the involvement of $\mathrm{My}$-Clp1 in tissue degeneration and the immune response. Nevertheless, a detailed functional characterization of the CLP in M. yessoensis is needed to further understand its molecular basis.

\section{ACKNOWLEDGMENTS}

Research supported by the National Ocean Public Welfare Scientific Research Project (under contract \#201305001).

\section{REFERENCES}

Arakane Y and Muthukrishnan S (2010). Insect chitinase and chitinase-like proteins. Cell Mol. Life Sci. 67: 201-216.

Arnold K, Bordoli L, Kopp J and Schwede T (2006). The SWISS-MODEL workspace: a web-based environment for protein structure homology modelling. Bioinformatics 22: 195-201.

Arocho A, Chen B, Ladanyi M and Pan Q (2006). Validation of the 2-DeltaDeltaCt calculation as an alternate method of data analysis for quantitative PCR of BCR-ABL P210 transcripts. Diagn. Mol. Pathol. 15: 56-61.

Badariotti F, Kypriotou M, Lelong C, Dubos M, et al. (2006). The phylogenetically conserved molluscan chitinase-like protein $1(\mathrm{Cg}-\mathrm{Clp} 1)$, homologue of human $\mathrm{HC}$-gp39, stimulates proliferation and regulates synthesis of extracellular 
matrix components of mammalian chondrocytes. J. Biol. Chem. 281: 29583-29596.

Badariotti F, Lelong C, Dubos M and Favrel P (2007). Characterization of chitinase-like proteins (Cg-Clp1 and Cg-Clp2) involved in immune defence of the mollusc Crassostrea gigas. FEBS J. 274: 3646-3654.

Badariotti F, Lelong C, Dubos M and Favrel P (2011). Identification of three singular glycosyl hydrolase family 18 members from the oyster Crassostrea gigas: Structural characterization, phylogenetic analysis and gene expression. Comp. Biochem. Phys. B 158: 56-63.

Bendtsen JD, Nielsen H, von Heijne G and Brunak S (2004). Improved prediction of signal peptides: SignalP 3.0. J. Mol. Biol. 340: 783-795.

Bleau G, Massicotte F, Merlen Y and Boisvert C (1999). Mammalian chitinase-like proteins. In: Chitin and Chitinases (Jollès P and Muzzarelli RAA, eds.). Birkhäuser Verlag, Basel, 211-221.

Burke RD (1983). The induction of metamorphosis of marine invertebrate larvae: stimulus and response. Can. J. Zool. 61: 1701-1719.

Fuhrman JA and Piessens WF (1985). Chitin synthesis and sheath morphogenesis in Brugia malayi microfilariae. Mol. Biochem. Parasit. 17: 93-104.

Giannetti N, Moyse E, Ducray A, Bondier JR, et al. (2004). Accumulation of Ym1/2 protein in the mouse olfactory epithelium during regeneration and aging. Neuroscience 123: 907-917.

Giard W, Lebel JM, Boucaud-Camou E and Favrel P (1998). Effects of vertebrate growth factors on digestive gland cells from the mollusc Pecten maximus L.: an in vitro study. J. Comp. Physiol. B 168: 81-86.

Goormachtig S, Van de Velde W, Lievens S, Verplancke C, et al. (2001). Srchi24, a chitinase homolog lacking an essential glutamic acid residue for hydrolytic activity, is induced during nodule development on Sesbania rostrata. Plant Physiol. 127: 78-89.

Han F, Zhang J, Li L, Zhang G, et al. (2012). Genetic diversity in two-generation of cultured Japanese scallop (Patinopecten (Mizuhopecten) yessoensis) populations. Mar. Sci. 36: 1-7. Abstract in English, written in Chinese.

He C, Yu H, Liu W, Su H, et al. (2012). A goose-type lysozyme gene in Japanese scallop (Mizuhopecten yessoensis): cDNA cloning, mRNA expression and promoter sequence analysis. Comp. Biochem. Physiol. B Biochem. Mol. Biol. 162: $34-43$.

Homer RJ, Zhu Z, Cohn L, Lee CG, et al. (2006). Differential expression of chitinases identify subsets of murine airway epithelial cells in allergic inflammation. Am. J. Physiol. Lung Cell Mol. Physiol. 291: L502-L511.

Houston DR, Recklies AD, Krupa JC and van Aalten DMF (2003). Structure and ligand-induced conformational change of the 39-kDa glycoprotein from human articular chondrocytes. J. Biol. Chem. 278: 30206-30212.

Johansen JS (2006). Studies on serum YKL-40 as a biomarker in diseases with inflammation, tissue remodelling, fibroses and cancer. Dan. Med. Bull. 53: 172-209.

Kawamura K, Shibata T, Saget O, Peel D, et al. (1999). A new family of growth factors produced by the fat body and active on Drosophila imaginal disc cells. Development 126: 211-219.

Kelleher TB, Mehta SH, Bhaskar R, Sulkowski M, et al. (2005). Prediction of hepatic fibrosis in HIV/HCV co-infected patients using serum fibrosis markers: The SHASTA index. J. Hepatol. 43: 78-84.

Kiefer F, Arnold K, Künzli M, Bordoli L, et al. (2009). The SWISS-MODEL Repository and associated resources. Nucleic Acids Res. 37: D387-D392.

Kneipp LF, Andrade AFB, de Souza W, Angluster J, et al. (1998). Trichomonas vaginalis and Tritrichomonas foetus: expression of chitin at the cell surface. Exp. Parasitol. 89: 195-204.

Lee CG, Da Silva CA, Lee J, Hartl D, et al. (2008). Chitin regulation of immune responses: an old molecule with new roles. Curr. Opin. Immunol. 20: 684-689.

Lee J, Takei K, Sakakibara H, Sun Cho H, et al. (2003). CHRK1, a chitinase-related receptor-like kinase, plays a role in plant development and cytokinin homeostasis in tobacco. Plant Mol. Biol. 53: 877-890.

Letunic I, Copley RR, Pils B, Pinkert S, et al. (2006). SMART 5: domains in the context of genomes and networks. Nucleic Acids Res. 34: D257-D260.

Li H, Fan J, Liu S, Yang Q, et al. (2012). Characterization of a myostatin gene (MSTN1) from spotted halibut (Verasper variegatus) and association between its promoter polymorphism and individual growth performance. Comp. Biochem. Phys. B Biochem. Mol. Biol. 161: 315-322.

$\mathrm{Li} \mathrm{Q}$, Xu K and Yu R (2007). Genetic variation in Chinese hatchery populations of the Japanese scallop (Patinopecten yessoensis) inferred from microsatellite data. Aquaculture 269: 211-219.

Li Y, Liu W, Gao X, Li W, et al. (2010). Construction of cDNA libraries from mantle and kidney of Japanese scallop (Mizuhopecten yessoensis) and ESTs analysis. J. Fish. Sci. China 17: 578-585. Abstract in English, written in Chinese.

Liu W, He C, Li W, Zhou Z, et al. (2010). Discovery of host defence genes in the Japanese scallop Mizuhopecten yessoensis Jay by expressed sequence tag analysis of kidney tissue. Aquac. Res. 41: 1602-1613. 
Mizoguchi E (2006). Chitinase 3-like-1 exacerbates intestinal inflammation by enhancing bacterial adhesion and invasion in colonic epithelial cells. Gastroenterology 130: 398-411.

Nagashima K, Sato M, Kawamata K, Nakamura A, et al. (2005). Genetic structure of Japanese scallop population in Hokkaido, analyzed by mitochondrial haplotype distribution. Mar. Biotechnol. 7: 1-10.

Ober C and Chupp GL (2009). The chitinase and chitinase-like proteins: a review of genetic and functional studies in asthma and immune-mediated diseases. Curr. Opin. Allergy Clin. Immunol. 9: 401-408.

Recklies AD, Ling H, White C and Bernier SM (2005). Inflammatory cytokines induce production of CHI3L1 by articular chondrocytes. J. Biol. Chem. 280: 41213-41221.

Shen T, Xu S, Yang M, Pang S, et al. (2011). Molecular cloning, expression pattern, and 3D structural analysis of the $M H C$ class IIB gene in the Chinese longsnout catfish (Leiocassis longirostris). Vet. Immunol. Immunopathol. 141: 33-45.

Tamura K, Dudley J, Nei M and Kumar S (2007). MEGA4: molecular evolutionary genetics analysis (MEGA) software version 4.0. Mol. Biol. Evol. 24: 1596-1599.

Thompson JD, Gibson TJ and Higgins DG (2002). Multiple sequence alignment using ClustalW and ClustalX. Curr. Protoc. Bioinformatics [doi: 10.1002/0471250953.bi0203s00].

Vogan CL, Costa-Ramos C and Rowley AF (2002). Shell disease syndrome in the edible crab, Cancer pagurus - isolation, characterization and pathogenicity of chitinolytic bacteria. Microbiology 148: 743-754.

Watanabe T, Kobori K, Miyashita K, Fujii T, et al. (1993). Identification of glutamic acid 204 and aspartic acid 200 in chitinase A1 of Bacillus circulans WL-12 as essential residues for chitinase activity. J. Biol. Chem. 268: 1856718572.

Wills-Karp M and Karp CL (2004). Chitin checking - novel insights into asthma. New. Engl. J. Med. 351: 1455-1457.

Zhu Q, Deng Y, Vanka P, Brown SJ, et al. (2004). Computational identification of novel chitinase-like proteins in the Drosophila melanogaster genome. Bioinformatics 20: 161-169. 